\title{
A myo-inositol diet for lung cancer prevention and beyond
}

\author{
Jae-il Roh, Han-Woong Lee \\ Department of Biochemistry, College of Life Science \& Biotechnology, Yonsei University, Seoul, Republic of Korea \\ Correspondence to: Han-Woong Lee. Department of Biochemistry, College of Life Science \& Biotechnology, Yonsei University, Seoul, Republic of \\ Korea. Email: hwl@yonsei.ac.kr. \\ Provenance: This is an invited Editorial commissioned by Executive Editor-in-Chief Jianxing He (Director of the Thoracic Surgery Department, The \\ First Affiliated Hospital of Guangzhou Medical University, Guangzhou, China). \\ Comment on: Unver N, Delgado O, Zeleke K, et al. Reduced IL-6 levels and tumor-associated phospho-STAT3 are associated with reduced tumor \\ development in a mouse model of lung cancer chemoprevention with myo-inositol. Int J Cancer 2018;142:1405-17.
}

Submitted Aug 16, 2018. Accepted for publication Aug 27, 2018.

doi: $10.21037 /$ jtd.2018.08.143

View this article at: http://dx.doi.org/10.21037/jtd.2018.08.143

Lung cancer is the deadliest among all the types of human cancer, causing approximately 160,000 cancer related deaths per year in the United States (1). The survival rate of patients diagnosed with lung cancer is poor, with an $80 \%$ mortality rate within a year of diagnosis. The most common type of lung cancer is non-small cell lung cancer (NSCLC), which often harbors a mutation in either KRAS (25\%), EGFR (25\%), or EML4-ALK (4\%) (2). Despite the increasing demand for drugs to treat lung cancer, efficient anti-NSCLC treatments have not yet been developed. Indeed, a targeted therapy for lung cancer patients carrying a $K R A S$ mutation is still unavailable. Recently, however, antibody-based inhibitors for immune checkpoints are showing an efficient survival benefit for lung cancer patients, though they are too expensive for regular clinical use and still present resistance with autoimmune responses (3).

Natural products from herbs, plants, foods, and their constituents have been used in traditional medicine to reduce disease incidence and adverse reactions to drugs. For instance, inositol and its derivatives support hormone utilization and improve mental health and female fertility $(4,5)$. A portion of previously known nutrients such as beta-carotene and carotenoids, as well as vegetables and fruits with high vitamin contents, can lower the risk of lung cancer by reducing the levels of reactive oxygen species and the detoxification of carcinogens $(6,7)$. Curcumin and ginger also show anti-tumorigenic effects through disruption of the cell cycle and induction of apoptosis $(8,9)$. In many cases, one of the major pathways involved in lung cancer, including AKT, NF-kB, MAPK, and STAT3, can be controlled by natural compounds with anti-tumorigenic effects (10).

Myo-inositol is a carbocyclic sugar that is naturally synthesized in some mammalian tissues from glucose and can act as an insulin-mimetic, resulting in lowered postprandial blood glucose (11). There have been reports on the benefits of the most abundant forms of inositol, myo-inositol and inositol hexaphosphate, including antitumorigenic properties, use as an adjuvant for conventional chemotherapy, and as a nutrient promoting psychological health $(5,12)$. In the August issue of the International fournal of Cancer, a team led by Edwin Ostrin, of the University of Texas MD Anderson Cancer Center, published their findings on the cancer preventive function of myo-inositol in KRAS mutation-induced lung tumorigenesis (13). The authors used a mouse model that specifically expresses mutant KRAS in the lung. Prior to the occurrence of evident premalignant lesions in the lung, 4-week-old mice began a diet including myo-inositol. This continued for 10 weeks and was followed by a measure of the tumor burden. Their results showed that the myo-inositol diet reduces the tumor burden in the mutant KRAS-induced lung cancer by up to $50 \%$ with no notable changes in body weight. Myo-inositol reduced the expression of mutant KRAS, while no significant change in the wild-type protein level was detected. Previous studies in humans have shown that KRAS activates the PI3K/AKT and MEK/ERK pathways and that myo-inositol inhibits PI3K signaling in patients $(14,15)$. Thus, researchers from Ostrin's group predicted a similar downregulation of that signaling in mice 
fed myo-inositol, however, neither PI3K/AKT signaling nor MEK/ERK signaling was affected by the myo-inositol diet. These results suggest a possible preventive effect of myo-inositol on mutant KRAS-driven lung cancer in mice, though further investigation is required to determine why pathways downstream from KRAS were not affected.

In a previous phase I clinical study in smokers in 2006, Stephen Lam's laboratory at the British Columbia Cancer Agency and the University of British Columbia, Canada showed that a daily dose of $18 \mathrm{~g}$ p.o. of myo-inositol for 3 months is safe with mild, if any, adverse effects. They reported a significant reduction in blood pressure and a regression of preexisting lesions in bronchial dysplasia (16). This study also revealed an increase in hemoglobin and complete response rate with a decrease in the progression rate, although statistical significance was only reached for the increase in hemoglobin level. These findings suggest a possible therapeutic effect of myo-inositol, not only in cancer and dysplasia but also in the circulatory system.

Ostrin's lab found an increase in the number of M1like macrophages $\left(\mathrm{CD} 45^{+} \mathrm{F} 4 / 80^{+} \mathrm{Ly} 6 \mathrm{c}^{-} \mathrm{MHCII}^{+}\right)$in bronchoalveolar lavage fluid from mice on the myo-inositol diet, but reported no changes in $\mathrm{T}$ cell infiltration or the population of $\mathrm{M} 2$-like macrophages $\left(\mathrm{CD} 45^{+} \mathrm{F} 4 / 80^{+} \mathrm{Ly} 6 \mathrm{c}^{-}\right.$ CD206 ${ }^{+}$). In addition, they detected increased IL-6 in normal lungs and a significant reduction of IL-6 level in the cancer bearing mice on the myo-inositol diet. The progression of KRAS mutation-driven lung cancer can be suppressed by a blockage of IL-6 $(17,18)$. IL-6 has dual functions in lung cancer, it can prevent the initiation but promote the progression, and myo-inositol may have a protective effect in both the initiation step, by increasing IL-6 in normal lung tissue, and the progression step, by decreasing IL-6 in lung cancer tissue, especially against the KRAS mutant type cancer. A clinical study conducted by Stephen Lam in 2016 revealed reduced IL-6 and PI3K activity in patients treated with myo-inositol (15). This reinforces Ostrin's findings that a diet including myoinositol results in alterations of IL-6 levels, even though PI3K activity was not changed in the KRAS mutant mice.

Currently, antibodies blocking immune-checkpoint pathways have been changing the therapeutic paradigm in several cancers, including lung cancer (19). Though there is a tremendous enhancement of survival rate following treatment with these inhibitors, many patients still suffer from primary and secondary immune resistances that render these treatments ineffective (20). Therefore, finding compounds that enhance the anti-tumorigenic efficacy of immune-checkpoint inhibitors (ICIs) would increase the number of positive patient outcomes. In one case, a combination of IL-6 inhibitors with ICIs has shown a reduction of hepatocellular carcinoma (21). Similar to the IL-6 inhibitors, a myo-inositol-mediated reduction of IL-6 secretion from macrophages could potentiate the efficacy of ICIs. Therefore, myo-inositol may protect and promote therapeutic efficiency against KRAS mutation-driven lung tumorigenesis through the regulation of IL-6 secretion in synergy with ICIs and alterations in the macrophage population present in the tumor microenvironment.

Myo-inositol is found in fruits, beans, grains, and nuts and has been used for the treatment of several diseases, including liver disease, depression, and neuropathy. Ostrin's study proposed an additional use for myo-inositol in the prevention and treatment of lung cancer. Its antitumorigenic effects might include a reduction of mutant KRAS expression, a change in the tumor microenvironment through the recruitment of polarized macrophages (M1-like) to the tumor sites, and the regulation of IL-6 secretion. However, there are still missing links to be solved, including (I) the absence of changes in PI3K/AKT and ERK activity despite the notable reduction of mutant KRAS expression, (II) the mechanism of how myo-inositol promotes the polarization of M1-like macrophages, and (III) whether its preventive effect against KRAS mutant lung cancer in mice can be recapitulated in humans. Therefore, many follow-up studies are required to reveal the mode-of-action mechanisms of myo-inositol and its potential role in both the prevention and treatment of lung cancer.

\section{Acknowledgements}

Funding: This work was supported by the National Research Foundation of Republic of Korea (grants 2015R1A2A1A01003845 and 2017R1A4A1015328).

\section{Footnote}

Conflicts of Interest: The authors have no conflicts of interest to declare.

\section{References}

1. Siegel RL, Miller KD, Jemal A. Cancer Statistics, 2017. CA Cancer J Clin 2017;67:7-30.

2. Bittner N, Ostoros G, Geczi L. New treatment options for lung adenocarcinoma--in view of molecular background. 
Pathol Oncol Res 2014;20:11-25.

3. Jhaveri KD, Perazella MA. Adverse Events Associated with Immune Checkpoint Blockade. N Engl J Med 2018;378:1163.

4. Regidor PA, Schindler AE. Myoinositol as a Safe and Alternative Approach in the Treatment of Infertile PCOS Women: A German Observational Study. Int J Endocrinol 2016;2016:9537632.

5. Levine J. Controlled trials of inositol in psychiatry. Eur Neuropsychopharmacol 1997;7:147-55.

6. Albanes D. Beta-carotene and lung cancer: a case study. Am J Clin Nutr 1999;69:1345S-50S.

7. Key TJ. Fruit and vegetables and cancer risk. Br J Cancer 2011;104:6-11.

8. Teiten MH, Eifes S, Dicato M, et al. Curcumin-the paradigm of a multi-target natural compound with applications in cancer prevention and treatment. Toxins (Basel) 2010;2:128-62.

9. Kim MO, Lee MH, Oi N, et al. [6]-shogaol inhibits growth and induces apoptosis of non-small cell lung cancer cells by directly regulating Akt1/2. Carcinogenesis 2014;35:683-91.

10. Zhong WL, Qin Y, Chen S, et al. Antitumor Effect of Natural Product Molecules against Lung Cancer. InTech, Rijeka 2017.

11. Croze ML, Soulage CO. Potential role and therapeutic interests of myo-inositol in metabolic diseases. Biochimie 2013;95:1811-27.

12. Vucenik I, Shamsuddin AM. Protection against cancer by dietary IP6 and inositol. Nutr Cancer 2006;5 5:109-25.

13. Unver N, Delgado O, Zeleke K, et al. Reduced IL-6 levels and tumor-associated phospho-STAT3 are associated with

Cite this article as: Roh JI, Lee HW. A myo-inositol diet for lung cancer prevention and beyond. J Thorac Dis 2018;10(Suppl 33):S3919-S3921. doi: 10.21037/jtd.2018.08.143 reduced tumor development in a mouse model of lung cancer chemoprevention with myo-inositol. Int J Cancer 2018;142:1405-17.

14. Zacchi P, Antonelli R, Cherubini E. Gephyrin phosphorylation in the functional organization and plasticity of GABAergic synapses. Front Cell Neurosci 2014;8:103.

15. Lam S, Mandrekar SJ, Gesthalter Y, et al. A Randomized Phase IIb Trial of myo-Inositol in Smokers with Bronchial Dysplasia. Cancer Prev Res (Phila) 2016;9:906-14.

16. Lam S, McWilliams A, LeRiche J, et al. A phase I study of myo-inositol for lung cancer chemoprevention. Cancer Epidemiol Biomarkers Prev 2006;15:1526-31.

17. Qu Z, Sun F, Zhou J, et al. Interleukin-6 Prevents the Initiation but Enhances the Progression of Lung Cancer. Cancer Res 2015;75:3209-15.

18. Caetano MS, Zhang H, Cumpian AM, et al. IL6 Blockade Reprograms the Lung Tumor Microenvironment to Limit the Development and Progression of K-ras-Mutant Lung Cancer. Cancer Res 2016;76:3189-99.

19. Velcheti V, Schalper K. Basic Overview of Current Immunotherapy Approaches in Cancer. Am Soc Clin Oncol Educ Book 2016;35:298-308.

20. Dammeijer F, Lau SP, van Eijck CH, et al. Rationally combining immunotherapies to improve efficacy of immune checkpoint blockade in solid tumors. Cytokine Growth Factor Rev 2017;36:5-15.

21. Liu H, Shen J, Lu K. IL-6 and PD-L1 blockade combination inhibits hepatocellular carcinoma cancer development in mouse model. Biochem Biophys Res Commun 2017;486:239-44. 